\title{
Thermal changes in Human Abdomen Exposed to Microwaves: A Model Study
}

\author{
Jagbir Kaur,"* and S.A. Khan ${ }^{2}$ \\ ${ }^{1}$ Department of Research and Development, I.K. Gujral Punjab Technical University, Kapurthala, Punjab, \\ India \\ ${ }^{2}$ Shiv Shankar Institute of Engineering and Technology, Patti, Tarn taran, Punjab, India \\ *corresponding author, E-mail: jagbirk@gmail.com
}

\begin{abstract}
The electromagnetic energy associated with microwave radiation interacts with the biological tissues and consequently, may produce thermo-physiological effects in living beings. Traditionally, Pennes' bioheat equation (BTE) is employed to analyze the heat transfer in biological medium. Being based on Fourier's Law, Pennes' BTE assumes infinite speed of propagation of heat transfer. However, heat propagates with finite speed within biological tissues, and thermal wave model of bioheat transfer (TWBHT) demonstrates this non-Fourier behavior of heat transfer in biological medium. In present study, we employed Pennes' BTE and TWMBT to numerically analyze temperature variations in human abdomen model exposed to plane microwaves at $2450 \mathrm{MHz}$. The numerical scheme comprises coupling of solution of Maxwell's equation of wave propagation within tissue to Pennes' BTE and TWMBT. Temperatures predicted by both the bioheat models are compared and effect of relaxation time on temperature variations is investigated. Additionally, electric field distribution and specific absorption rate (SAR) distribution is also studied. Transient temperatures predicted by TWMBT are lower than that by traditional Pennes' BTE, while temperatures are identical in steady state. The results provide comprehensive understanding of temperature changes in irradiated human body, if microwave exposure duration is short.
\end{abstract}

\section{Introduction}

Microwaves are widely utilized in a number of applications including RADAR, communication, medical machines, navigation equipment, microwave oven and industrial heating devices [1-3]. Increasing use of microwaves has attracted researchers to quantify the health risks due to microwave exposure. International safety agencies such as ICNIRP, IEEE, FCC, etc. have set safety standards for microwave radiation exposure in terms of specific absorption rate (SAR), which is the measure of the rate of electromagnetic (EM) energy absorbed by the body exposed to radiation [4-6]. The biological effects caused by absorption of EM energy depend upon intensity, frequency, waveform and duration of exposure [7]. The EM energy carried by microwaves is transferred to the biological tissue, which results in ion acceleration and collisions among molecules. Consequently, the local temperature of the tissue rises [8]. An increase of $1-5^{\circ} \mathrm{C}$ in human body can cause many malformations including male infertility, brain lesions, and blood chemistry changes [9]. Even a very small temperature change of $0.2-0.5^{\circ} \mathrm{C}$ may cause altered thermoregulatory responses [10]. Considering the hazards of temperature rise in biological tissue, it is important to assess the temperature alterations in human body caused by microwave exposure.

Many researchers have investigated temperature rise within various biological tissues exposed to electromagnetic radiation using Pennes' bioheat transfer equation (BTE) [1114]. Gandhi et al. determined the temperature elevation in pinna and brain exposed to radiofrequency (RF) radiation [15]. Wessapan et al. employed BTE to study temperature rise in brain, eye and human trunk due to RF waves exposure [16-18]. Pennes' BTE assumes that the thermal wave propagates with infinite speed inside the tissue, and any thermal disturbance in the tissue is instantaneously felt by the body $[19,20]$. However, in heterogeneous biological media such as human body, relaxation time $(\tau)$ is required for accumulation of enough energy to transfer it to the nearest element $[21,22]$. Thermal wave model of bioheat transfer (TWMBT) incorporates relaxation time to account for the lag time it takes for the EM wave to respond to heat propagation through biological tissue $[23,24]$. Few studies employed TWMBT to determine the risk that electromagnetic waves have when skin tissues are exposed to them [19, 22-25]. Data reporting the value of relaxation time for heat transfer in living beings is limited in the literature. Xu et al. [26] examined heat transfer in skin tissue with relaxation time range $0-15 \mathrm{~s}$, while some experimental studies have shown that relaxation time ranges from 15 to 30 s [27-29].

In present study, we have investigated the temperature variations in human abdomen exposed to plane microwave. Previously, Wessapen et al. [18] employed Pennes' BTE to determine thermal variations in human trunk, but they did not consider relaxation time in their analysis. Relaxation time is a significant parameter for heat propagation in heterogeneous biological tissues, since it characterizes the interaction of inner structural elements of non-homogeneous medium during process of heat transfer [30]. In our analysis, 
we have included both Pennes' BTE and TWMBT model to study temperature distribution and effect of relaxation time on the heat propagation within the human abdominal cavity. A two-dimensional vertical cross sectional model of human abdomen is used for numerical simulation using finite element method (FEM). The frequency of microwave exposure is $2450 \mathrm{MHz}$ and power densities taken for analysis are the safety limits set by ICNIRP i.e. $1 \mathrm{~mW} \mathrm{~cm}^{-2}$ for general population and $5 \mathrm{~mW} \mathrm{~cm}^{-2}$ for the occupational population [4]. Although, most applications utilize low power microwaves, but heating and drying industry and military applications use high powers [18]. The leakage or accidental exposure to high power microwaves may be hazardous, therefore, power density of $50 \mathrm{~mW} \mathrm{~cm}$ is also considered in investigation. The electric field distribution, SAR and temperature variations are numerically analyzed in this study.

\section{Mathematical models}

\subsection{Pennes' bioheat transfer equation (BTE)}

Pennes' BTE is the pioneer mathematical model for analyzing heat conduction in biological tissues. It is expressed as:

$$
\rho C \frac{\partial T}{\partial t}=\nabla \cdot(k \nabla T)+\rho_{b} C_{b} \omega_{b}\left(T_{b}-T\right)+Q_{m}+Q_{r}
$$

Where $\rho, C$ and $k$ are the density $\left(\mathrm{kg} \mathrm{m}^{-3}\right)$, specific heat ( $\mathrm{J}$ $\mathrm{kg}^{-1} \mathrm{~K}^{-1}$ ), and the thermal conductivity $\left(\mathrm{W} \mathrm{m}^{-1} \mathrm{~K}^{-1}\right)$ of tissue, respectively. $\rho_{b}$ and $C_{b}$ are the density $\left(\mathrm{kg} \mathrm{m}^{-3}\right)$ and specific heat $\left(\mathrm{J} \mathrm{kg}^{-1} \mathrm{~K}^{-1}\right)$ of the blood, respectively. $\omega_{b}$ is the blood perfusion rate $\left(\mathrm{s}^{-1}\right) ; T$ and $T_{b}$ are tissue and blood temperatures $\left({ }^{\circ} \mathrm{C}\right)$, respectively. $Q_{m}$ is metabolic heat generation in the tissue $\left(\mathrm{W} \mathrm{m}^{-3}\right)$ and $Q_{r}$ is the external heat source term (electromagnetic heat-source density) $\left(\mathrm{W} \mathrm{m}^{-3}\right)$. The term $\nabla .(k \nabla T)$ in Equation (1) represents the heat conduction inside the human body and is based on Fourier's law:

$$
q(r, t)=-k \nabla T(r, t)
$$

This law implies that any thermal disturbance is instantaneous within the body [26]. The term $\rho_{b} C_{b} \omega_{b}\left(T_{b}-\right.$ $T$ ) describes the heat caused by convection inside the body. $Q_{r}$ represents the resistive heat generated by the electromagnetic source and is expressed as [16]:

$$
Q_{r}=\frac{1}{2} \sigma|E|^{2}
$$

where $\sigma$ is the conductivity $\left(\mathrm{S} \mathrm{m}^{-1}\right)$ of the tissue and $E$ is the electric field $\left(\mathrm{V} \mathrm{m}^{-1}\right)$ within the tissue exposed to microwaves. $Q_{m}$ is neglected and assumed zero in the analysis, since we aim to evaluate the effect of electromagnetic energy associated with microwaves only . As the resistive heat generated by the microwave exposure ( $Q_{r}$ ) propagates through the human body, the electromagnetic energy is absorbed by the tissues. The energy absorbed is denoted in terms of SAR as [31]:

$$
S A R=\sigma|E|^{2} / \rho
$$

\subsection{Thermal Wave Model of Bioheat Transfer (TWMBT)}

Fourier's law defined by Equation (2), successfully explains the heat conduction in homogeneous materials [30]. However, non-homogeneous structures such as biological mediums exhibit a higher microscale thermal response. Therefore, a non-Fourier model should be considered for heat propagation in biological tissues [32]. The concept of thermal lag is formulated in the Fourier's law with addition of relaxation time ' $\tau$ ' in Equation (2) [33-34].

$$
q(r, t+\tau)=-k \nabla T(r, t)
$$

where $\tau=\alpha / C_{t}^{2}$ is defined as the thermal relaxation time with $\alpha$ being the thermal diffusivity $\left(\mathrm{m}^{2} \mathrm{~s}^{-1}\right)$, and $\mathrm{C}_{\mathrm{t}}$ being the speed of thermal wave in the medium $\left(\mathrm{m} \mathrm{s}^{-1}\right)$ [24]. Substituting the first order Taylor expansion of Equation (5) in Equation (1), a new TWMBT is obtained:

$$
\begin{aligned}
\rho C \tau \frac{\partial^{2} T}{\partial t^{2}}=k \nabla^{2} T & -\rho_{b} C_{b} \omega_{b} T-\left(\tau \rho_{b} C_{b} \omega_{b}+\rho C\right) \frac{\partial T}{\partial t} \\
& +\rho_{b} C_{b} \omega_{b} T_{b}+Q_{m}+Q_{r}+\tau \frac{\partial Q_{m}}{\partial t} \\
& +\tau \frac{\partial Q_{r}}{\partial t}
\end{aligned}
$$

Since $Q_{m}$ is assumed zero in our analysis, Equation (6) can be re-written as:

$$
\begin{aligned}
\rho C \tau \frac{\partial^{2} T}{\partial t^{2}}=k \nabla^{2} T & -\rho_{b} C_{b} \omega_{b} T-\left(\tau \rho_{b} C_{b} \omega_{b}+\rho C\right) \frac{\partial T}{\partial t} \\
& +\rho_{b} C_{b} \omega_{b} T_{b}+Q_{r}+\tau \frac{\partial Q_{r}}{\partial t}
\end{aligned}
$$

Due to presence of two double derivative terms in Equation (7), TWMBT is also known as hyperbolic model of heat transfer.

\section{Numerical Method}

A two dimensional vertical cross sectional model of human abdomen (Fig. 1) is obtained by reconstructing the human model used by Shiba and Higaki [35]. Vector graphics editor INKSCAPE is utilized to reconstruct the abdomen model. This is an anatomically based human body model, which contains 9 abdominal organs. The thermal and dielectric properties of different organs are listed in Table 1. The Model is exposed to transverse electric (TE) plane wave of frequency $2450 \mathrm{MHz}$, propagating in positive $\mathrm{X}$ direction with a specific power density. For numerical analysis, FEM based simulation software COMSOL $^{\mathrm{TM}}$ Multiphysics is used. The investigation is executed in two steps, i.e. 
propagation of electromagnetic waves and bioheat transfer analysis inside the body.

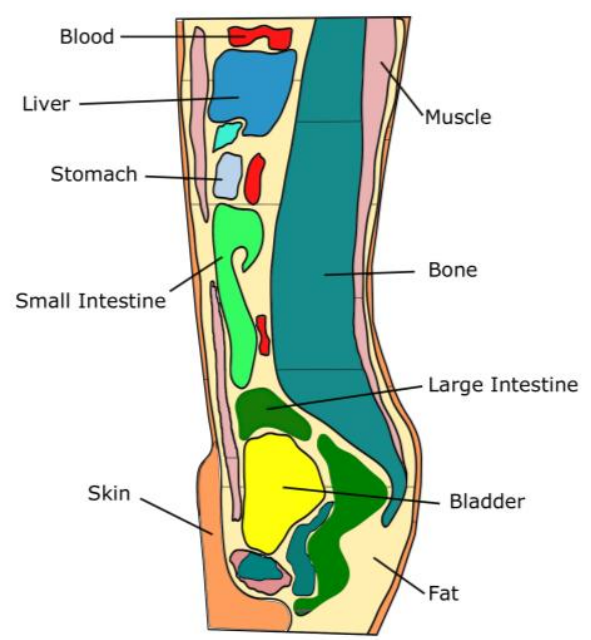

Figure 1: Vertical cross section of human abdomen obtained from Shiba and Higaki [35]

\subsection{Propagation of electromagnetic waves}

Maxwell's equations govern the propagation of electromagnetic waves inside the human body and electric field intensity (E) can be determined by equation:

$$
\nabla \times \frac{1}{\mu_{r}}(\nabla \times E)-k_{0}^{2}\left(\varepsilon_{r}-\frac{j \sigma}{\omega \varepsilon_{0}}\right) E=0
$$

where $\mu_{r}$ is relative magnetic permeability, $\varepsilon_{r}$ is relative permittivity, $\varepsilon_{0}=8.8542 \times 10^{-12} \mathrm{~F} \mathrm{~m}^{-1}, k_{0}$ is the free space wave number $\left(\mathrm{m}^{-1}\right)$ and $\omega$ is angular frequency $(\mathrm{Hz})$. Using the value of $E$ at any point, local SAR can be computed at that point by utilizing Equation (4). In electromagnetic analysis, scattering boundary condition is applied on outer edges of the calculated domain (Fig. 2), as

$$
\begin{aligned}
n \times(\nabla \times E)-j k n \times & (E \times n) \\
= & -n \times\left(E_{0} \times j k(n-k)\right) \exp (-j k . r)
\end{aligned}
$$

where $k$ is the wave number $\left(\mathrm{m}^{-1}\right), \mathrm{n}$ is normal vector,

\begin{tabular}{|c|c|c|c|c|c|c|}
\hline & $\begin{array}{l}\text { Thermal } \\
\text { conductivity, } \\
\mathrm{k} \\
\left(\mathrm{W} \mathrm{m}^{-1} \mathrm{~K}^{-1}\right)\end{array}$ & $\begin{array}{l}\text { Specific heat, } \\
\mathrm{C} \quad\left(\mathrm{J} \mathrm{kg}^{-1} \mathrm{~K}^{-}\right. \\
\left.{ }^{1}\right)\end{array}$ & $\begin{array}{l}\text { Density, } \rho \\
\left(\mathrm{kg} \mathrm{m}^{-3}\right)\end{array}$ & $\begin{array}{l}\text { Blood } \\
\text { perfusion } \\
\text { rate, } \omega_{\mathrm{b}}\left(\mathrm{s}^{-1}\right)\end{array}$ & $\begin{array}{l}\text { Conductivity, } \\
\sigma \\
(\mathrm{S} / \mathrm{m})\end{array}$ & $\begin{array}{l}\text { Relative } \\
\text { permittivity, } \\
\varepsilon\end{array}$ \\
\hline Bladder $^{\mathrm{a}}$ & 0.56 & 3900 & 1040 & 0 & 0.68532 & 18.001 \\
\hline Blood & 0.52 & 3617 & 1050 & -- & 2.54 & 58.3 \\
\hline Bone & 0.32 & 1313 & 1908 & $4.36^{*} 10^{-04}$ & 0.394 & 11.4 \\
\hline Fat & 0.21 & 2348 & 911 & $4.58 * 10^{-04}$ & 0.10452 & 5.2801 \\
\hline $\begin{array}{l}\text { Large } \\
\text { intestine }\end{array}$ & 0.54 & 3655 & 1088 & $1.39 * 10^{-02}$ & 2.0383 & 53.879 \\
\hline Liver & 0.52 & 3540 & 1079 & $1.7201 * 10^{-02}$ & 1.6864 & 43.035 \\
\hline Muscle & 0.49 & 3421 & 1090 & $8.69 * 10^{-03}$ & 1.7388 & 52.729 \\
\hline Skin & 0.37 & 3437 & 1109 & 0.02 & 1.464 & 38.007 \\
\hline $\begin{array}{l}\text { Small } \\
\text { intestine }\end{array}$ & 0.49 & 3653 & 1030 & $1.74 * 10^{-02}$ & 3.1731 & 54.425 \\
\hline Stomach & 0.53 & 3553 & 1088 & $7 * 10^{-03}$ & 2.2105 & 62.158 \\
\hline
\end{tabular}
$j=\sqrt{-1}$, and $E_{0}$ is the incident plane wave $\left(\mathrm{V} \mathrm{m}^{-1}\right)$.

Table 1 : Thermal and dielectric properties of tissues at $2450 \mathrm{MHz}[36,37]$

a[18] 


\subsection{Bioheat transfer analysis}

The temperature distribution within the human trunk is determined using Pennes' BTE (Equation (1)) and TWMBT (Equation (7)). Both the equations are coupled to the solution of Maxwell's equation. Substituting values of E, $Q_{r}$ is calculated (using Equation (3)) and temperature rise is determined using Equation (1) and Equation (7).

The temperature of human body is assumed uniform and its initial value is $37^{\circ} \mathrm{C}$. Therefore, initial condition for bioheat transfer is:

$\mathrm{T}=37^{\circ} \mathrm{C}$ at $\mathrm{t}=0$ and $\frac{\partial T}{\partial t}=0$ at $\mathrm{t}=0$

No heat is exchanged between the calculated domain and the outer region. Hence, thermal insulation boundary condition is employed on the outer boundaries of the model (Fig. 2).

n. $(k \nabla T)=0$

\subsection{Procedure for simulation}

Finite element method (FEM) is used for numerical simulation, since FEM models provide quick and accurate solution for the system of multiple differential equations [38]. The electromagnetic heat source term $\left(Q_{r}\right)$ of heat transfer equations (Equation (1) and Equation (7)) is determined by using the electromagnetic calculations.

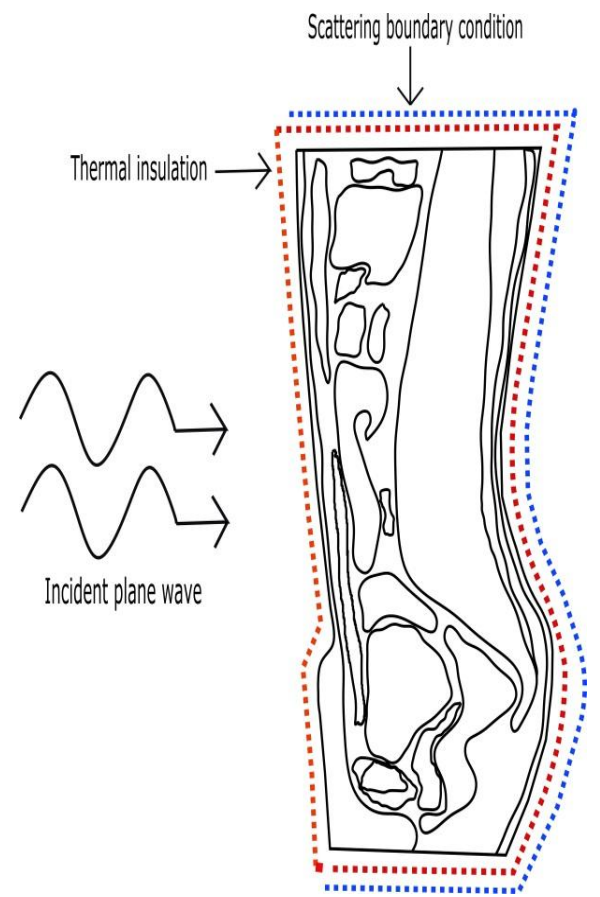

Figure 2: Boundary conditions for numerical

analysis
The temperature at every point within domain is calculated using electromagnetic field at that point and the steps are repeated until the steady state of temperature is reached. The domain is discretized using tetrahedral elements with a total number of 101805 elements (Fig. 3a). A mesh convergence test is performed to approximate the number of elements above which the solution is independent of mesh elements (Fig. 3b). The mesh independence is attained for the number of elements more than 60000 . Higher numbers of elements are not tested due to lack of computational memory and performance.

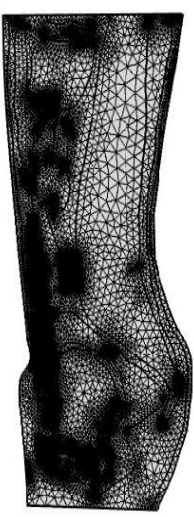

(a)

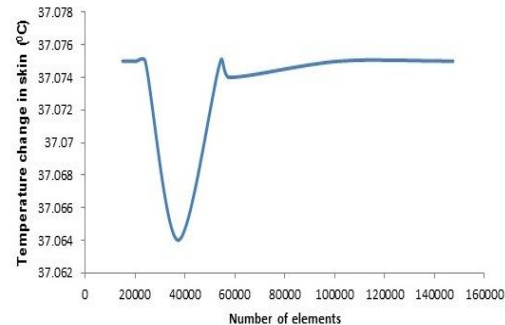

(b)
Figure 3: (a) Tetrahedral mesh and (b) mesh convergence for FEM analysis

\section{Results}

Electromagnetic and temperature variations are evaluated in the human abdomen exposed to plane wave of microwave frequency. The frequency of the exposure is $2450 \mathrm{MHz}$ and power densities are $1 \mathrm{~mW} \mathrm{~cm}^{-2}, 5 \mathrm{~mW} \mathrm{~cm}^{-2}$, and $50 \mathrm{~mW} \mathrm{~cm}^{-}$ ${ }^{2}$. First two power densities mentioned are the exposure limits set by ICNIRP for general and occupational population respectively, and a special case of leakage radiation from high power microwave devices with power density $50 \mathrm{~mW} \mathrm{~cm}$ is considered. The electric field distribution is determined using Maxwell's equation, while TWMBT and Pennes' BTE are employed to investigate the temperature distribution within human body. In TWMBT, the relaxation time of $0 \mathrm{~s}, 10 \mathrm{~s}, 20 \mathrm{~s}$ and $30 \mathrm{~s}$ is used in analysis.

\subsection{Validation}

Measuring the electric field and temperature distribution directly inside human body to validate the numerical solution is not possible due to ethical considerations. To 
validate the accuracy of numerical simulation used in this study, a simple case of simulated results is verified against the results with the human trunk model studied by Nishizawa and Hashimoto [39]. They have analyzed local SAR in a three-layer human abdomen model exposed to plane microwaves at $1300 \mathrm{MHz}$ and power density $1 \mathrm{~mW}$ $\mathrm{cm}^{-2}$. Fig. 4a shows two-dimensional schematic geometry of human abdomen used in validation case. The model consists of three layers viz. skin, fat and muscle. The plane wave $\mathrm{E}$ (TM) of frequency $1300 \mathrm{MHz}$ and power density $1 \mathrm{~mW} \mathrm{~cm}^{-}$ ${ }^{2}$, propagating along positive $\mathrm{X}$-direction is incident on left side of model. In validation test case, we have set the exposure conditions as employed by Nishizawa and Hashimoto. The local SAR is plotted along cross sectional line through the abdomen; the graph (Fig. 4b) shows good agreement in SAR values between present solution and that of Nishizawa and Hashimoto. Small deviations occurring between two results may be due to different numerical scheme used and input dielectric properties of the tissues. This encouraging comparison gives confidence in accuracy of the simulation used in present study.

\subsection{Electric field and SAR distribution}

Fig. 5 illustrates the electric field distribution within the exposed human body. The maximum electric field is predicted beneath the exposed surface of skin and its magnitude decreases rapidly with distance from the surface.
This behavior of electric field can be result of small wavelength and hence, small penetration depth of microwaves. Therefore, electric field does not penetrate deep inside the body. The power of microwaves influences the electric field significantly; for greater values of power densities, electric field has higher values. The maximum electric field calculated is $46.062 \mathrm{~V} \mathrm{~m}^{-1}, 104.67 \mathrm{~V} \mathrm{~m}^{-1}$ and $330.82 \mathrm{~V} \mathrm{~m}^{-1}$ for exposure power densities $1 \mathrm{~mW} \mathrm{~cm}^{-2}, 5$

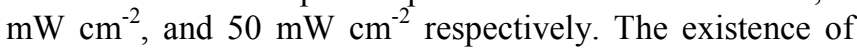
electric field within body conveys that EM energy is deposited in the tissues. The energy absorbed by the tissues is expressed in terms of SAR.

Fig. 6 shows local SAR distribution within the exposed human trunk. Since SAR at a point is proportional to the electric field at that point, local SAR distribution pattern is similar to the electric field distribution. The maximum values of SAR are obtained in the skin layer for all the incident power densities; $\mathrm{SAR}_{\max }$ is $2.895 \mathrm{~W} \mathrm{~kg}^{-1}, 14.447 \mathrm{~W}$ $\mathrm{kg}^{-1}$ and $144.45 \mathrm{~W} \mathrm{~kg}^{-1}$ for power density $1 \mathrm{~mW} \mathrm{~cm}^{-2}, 5 \mathrm{~mW}$ $\mathrm{cm}^{-2}$, and $50 \mathrm{~mW} \mathrm{~cm}$ respectively. The safety limits on local SAR within trunk set by ICNIRP for general and occupational population are $2 \mathrm{~W} / \mathrm{kg}$ and $10 \mathrm{~W} / \mathrm{kg}$, respectively; the predicted $\mathrm{SAR}_{\max }$ values have exceeded these limits in all the exposure cases.

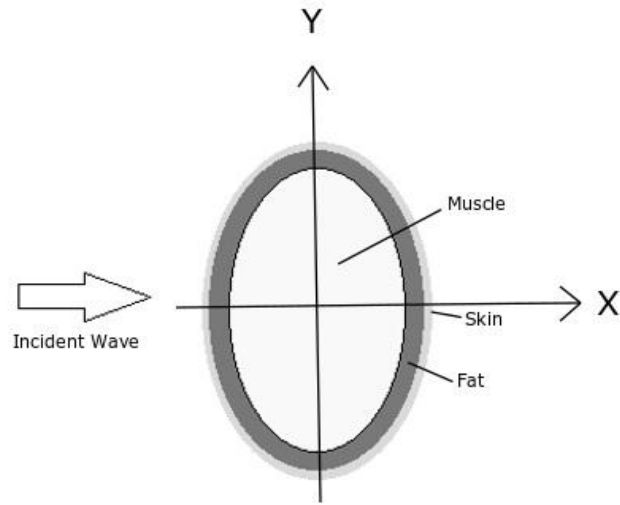

(a)

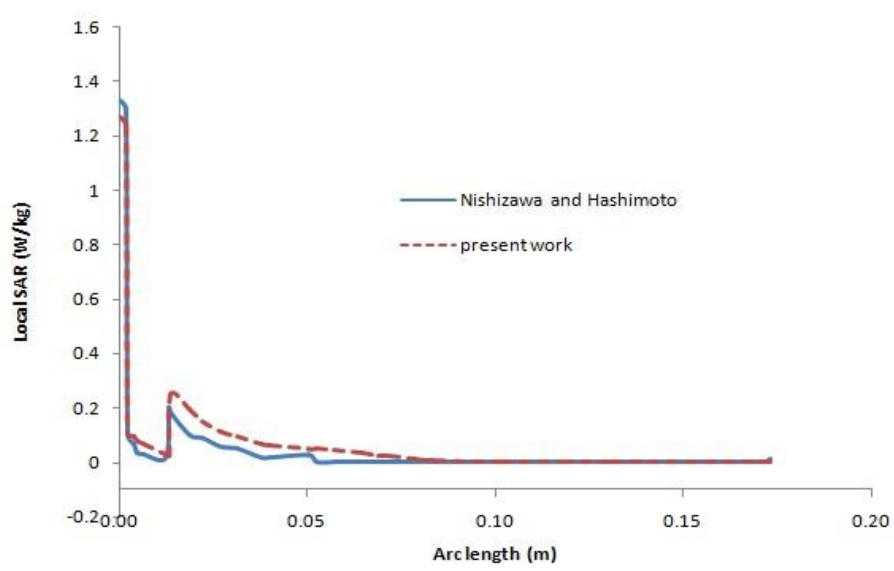

(b)

Figure 4: (a) 2-dimensional geometry for validation of model from Nishizawa and Hashimoto [39] (b) comparison of local SAR distribution calculated by present numerical simulation and that by Nishizawa and Hashimoto 


\subsection{Temperature distribution}

The temperature variations are analyzed using Pennes' BTE and TWMBT. The transient temperature predictions by both bioheat equations are significantly different (Fig. 9). When steady state is reached, both the equations produce similar results. Fig. 7 portrays the steady state temperature distribution inside human body. Since most of the energy carried by microwaves is absorbed in skin layer, maximum temperature rise is in the skin layer of abdomen. Maximum temperature change is $0.015{ }^{\circ} \mathrm{C}, 0.073{ }^{\circ} \mathrm{C}$ and $0.735{ }^{\circ} \mathrm{C}$ for power density $1 \mathrm{~mW} \mathrm{~cm}{ }^{-2}, 5 \mathrm{~mW} \mathrm{~cm}^{-2}$, and $50 \mathrm{~mW} \mathrm{~cm}^{-2}$ respectively.

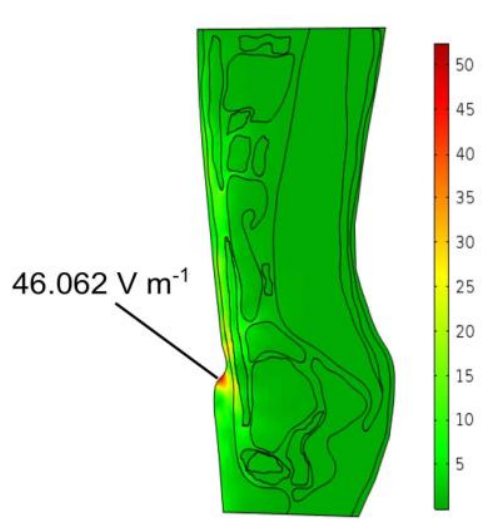

(a)

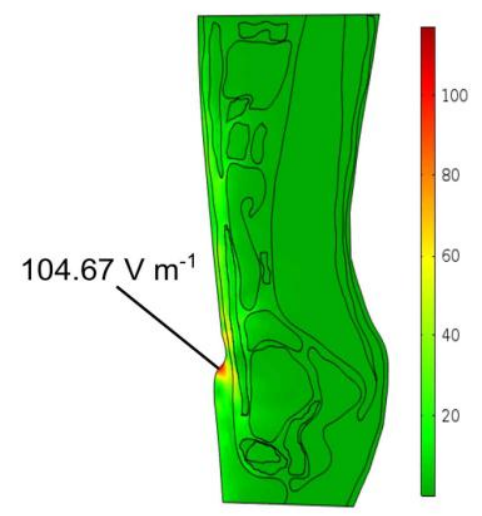

(b)

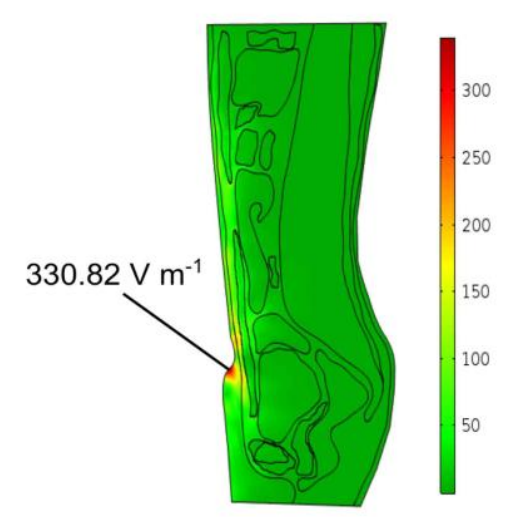

(c)

Figure 5: Electric field distribution in human trunk exposed to $2450 \mathrm{MHz}$ plane wave at (a) $1 \mathrm{~mW} \mathrm{~cm}{ }^{-2}$, (b) $5 \mathrm{~mW}$ $\mathrm{cm}^{-2}$ and (c) $50 \mathrm{~mW} \mathrm{~cm}^{-2}$

Relaxation time ' $\tau$ ' is an important parameter of TWMBT, which is time needed for accumulating the heat energy required for its transfer to the nearest element within the non-homogeneous body. In present analysis, the temperature deviations are studied for $\tau$ values $0 \mathrm{~s}, 10 \mathrm{~s}, 20 \mathrm{~s}$ and $30 \mathrm{~s}$. Temperature changes are investigated at the point within the skin where maximum change in temperature has occurred (Fig. 8). To study the changes in temperature along horizontal distance within the abdomen, an extrusion line passing through five organs is considered (Fig. 8).

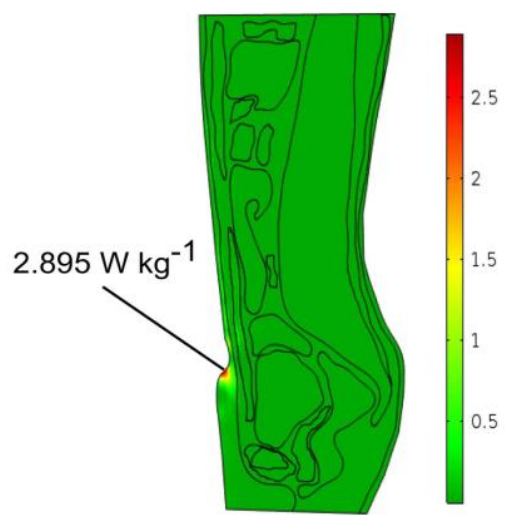

(a)

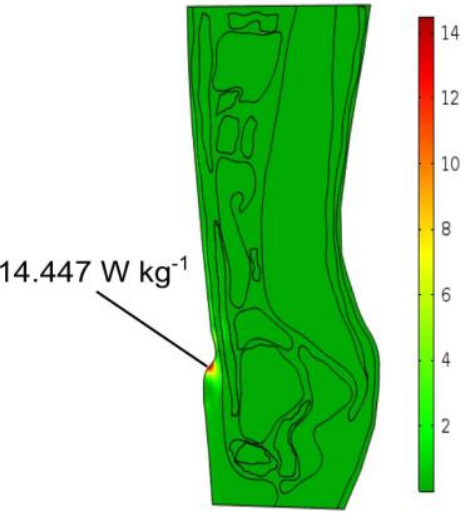

(b)

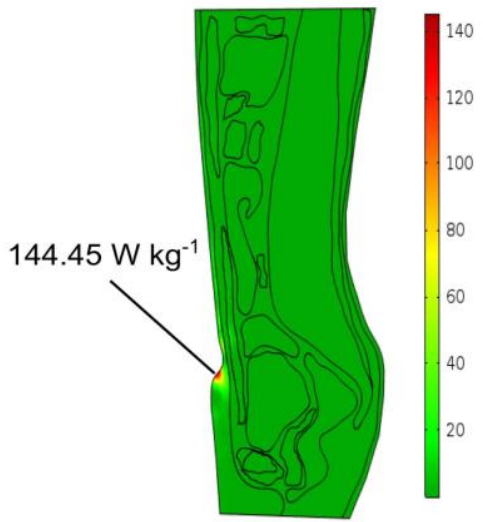

(c)

Figure 6: SAR distribution in human trunk exposed to $2450 \mathrm{MHz}$ plane wave at (a) $1 \mathrm{~mW} \mathrm{~cm}^{-2}$, (b) $5 \mathrm{~mW} \mathrm{~cm}^{-2}$ and (c) $50 \mathrm{~mW} \mathrm{~cm}^{-2}$ 


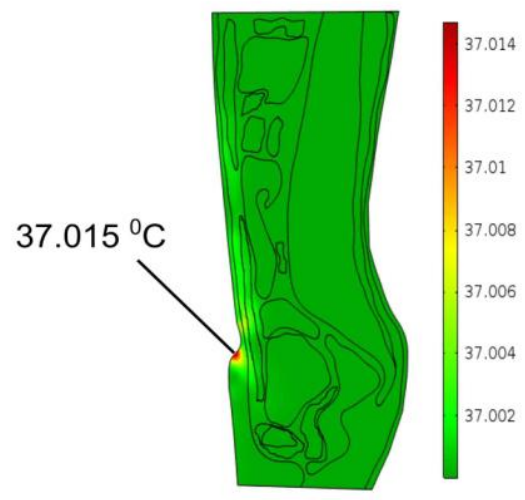

(a)

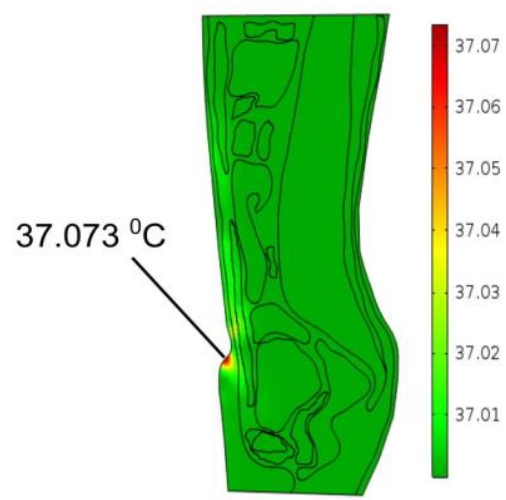

(b)

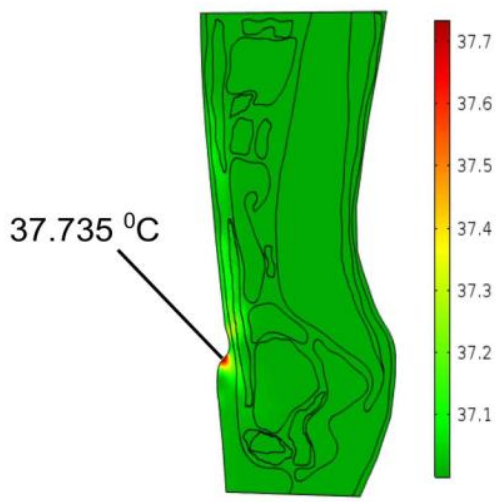

(c)

Figure 7: Steady state temperature distribution in the human trunk exposed to $2450 \mathrm{MHz}$ plane wave at (a) $1 \mathrm{~mW} \mathrm{~cm}^{-2}$, (b) 5 $\mathrm{mW} \mathrm{cm} \mathrm{cm}^{-2}$ and (c) $50 \mathrm{~mW} \mathrm{~cm}^{-2}$

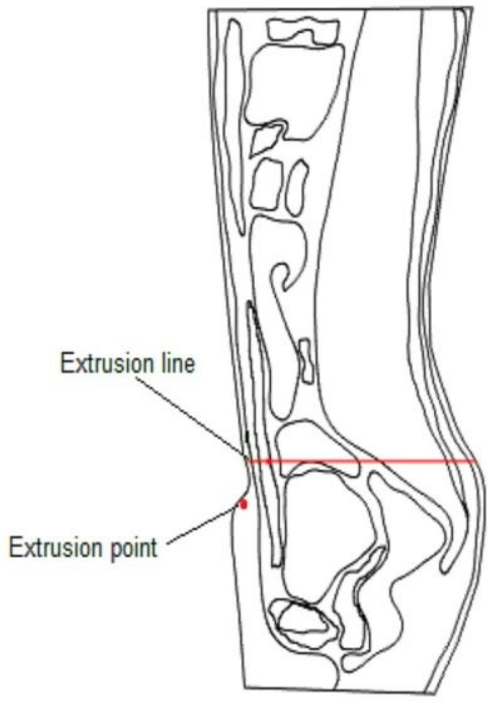

Figure 8: Extrusion line and point where temperature variations are analyzed

Fig. 9 shows the temperature variations w.r.t. duration of exposure for different values of relaxation time. These changes are studied at extrusion point for exposure power densities of $1 \mathrm{~mW} \mathrm{~cm}^{-2}, 5 \mathrm{~mW} \mathrm{~cm}$, and $50 \mathrm{~mW} \mathrm{~cm}^{-2}$. The temperature profiles predicted by Pennes' BTE and TWMBT $(\tau=0$ s) are identical for all power densities, which implies that for relaxation time, $\tau=0 \mathrm{~s}$, both thermal models yield same temperatures. Therefore, Pennes' BTE can be considered as a special case of TWMBT.

Pennes' BTE and TWMBT predict different transient temperatures; however, the steady state temperatures predicted by both the models are identical. Temperature curves estimated by Pennes' BTE and TWMBT with $\tau=0 \mathrm{~s}$ reach the steady state more quickly than the curves predicted by larger relaxation times. In each exposure case, all the temperature curves merge in steady state. During the transient state, temperature estimated by Pennes' BTE is highest, which is same as that by TWMBT with $\tau=0 \mathrm{~s}$. The greater values of relaxation time $(\tau)$ predict lower temperatures; lowest temperature curve is projected by TWMBT with $\tau=30 \mathrm{~s}$. It is also clear from Fig. 9 that temperature rises more quickly for smaller relaxation time values than the temperatures predicted with larger relaxation times. The difference between the estimated temperatures decreases as the exposure duration increases. At $30 \mathrm{~s}$ of exposure the temperaure precicted by Pennes' model is greater than that predicted by TWMBT with $\tau=30$ s by $250 \%$ and at $60 \mathrm{~s}$, this difference reduces to $135 \%$, finally at around $300 \mathrm{~s}$ of exposure the difference in the predicted temperatures vanishes. This result is especially significant for higher power densities if exposure duration is short, because transient temperatures differ considerably for different values of relaxation time Since, relaxation time characterizes the heterogeneous structure of human body; 
the temperature transitions predicted by TWMBT may be more realistic than that by Pennes' BTE.

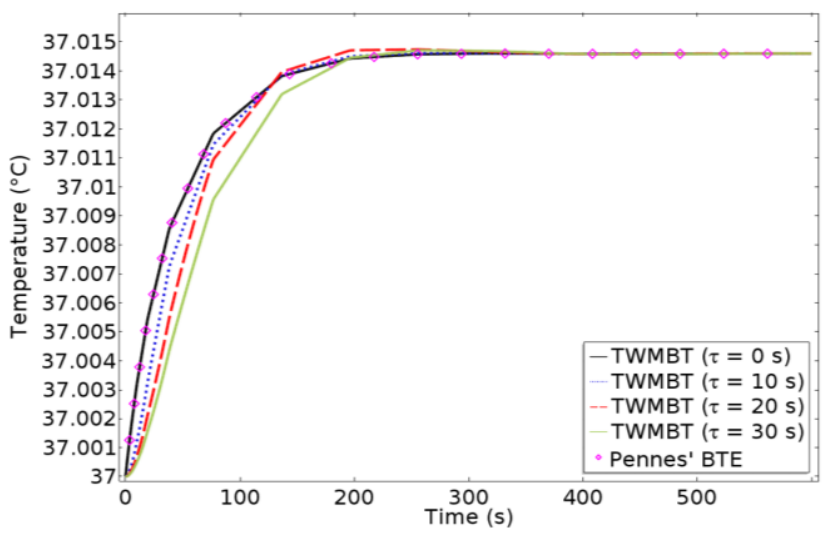

(a)

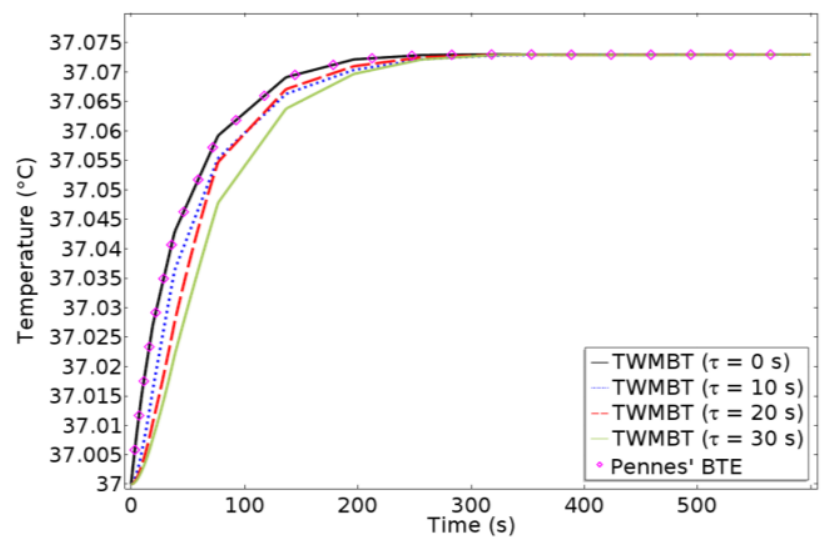

(b)

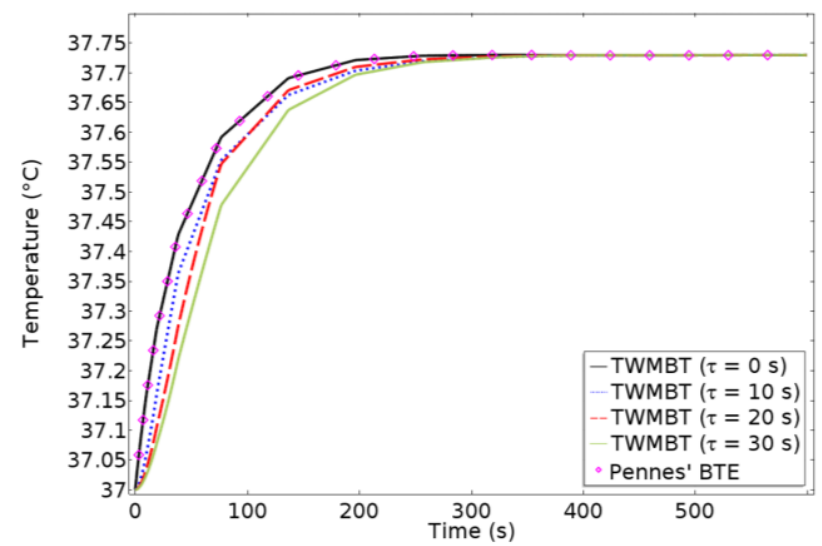

(c)

Figure 9: Variations in maximum temperature in skin versus time at (a) $1 \mathrm{~mW} \mathrm{~cm}^{-2}$, (b) $5 \mathrm{~mW} \mathrm{~cm}^{-2}$ and (c) $50 \mathrm{~mW} \mathrm{~cm}^{-2}$

Fig. 10 portrays the temperature distributions at 1 minute of microwave exposure for various power densities along the extrusion line. The extrusion line runs through skin, fat, muscle, large intestine and bone within human trunk. Temperature curves for Pennes' BTE and TWMBT with $\tau=0 \quad \mathrm{~s}$ overlap, and predict highest temperature along extrusion line.

Steep temperature rise occurs in skin layer; and maximum temperature is observed at skin-fat interface. The temperature decreases sharply with linear distance along the extrusion line. Greater values of relaxation time predict lower temperatures. It is observed that the difference between temperature profiles is more near skin surface, it decreases as distance from surface increases and finally the difference vanishes and curves merge at around $0.035 \mathrm{~m}$ away from exposed surface. Since the abdomen model is non-homogeneous, small peaks in temperature curves may be due to heterogeneous structure of considered domain. Fig. 11 shows the temperature distribution at different exposure times determined by Pennes' BTE along extrusion

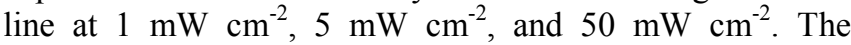
temperature rises as exposure time increases, the rise is trivial after $300 \mathrm{~s}$ of exposure and steady state is achieved at $540 \mathrm{~s}$ of microwave exposure. At $30 \mathrm{~s}$ of exposure, the highest temperature peak exists in skin layer. As the time progresses, peak is shifting from skin layer towards fat layer. This behavior indicates onset of blood perfusion with time, which transmits the heat from skin to fat layer. In addition, the small peaks in temperature curves smoothen as time advances; only major peak at skin-fat interface is present in steady state curve. The reason for disappearance of minor temperature peaks within muscle layer may be blood perfusion in muscle, which sweeps the heat accumulated, as time passes. Very low blood perfusion rate of fat layer $\left(4.58 \times 10^{-04}\right)$ may be held responsible for presence of temperature peak in this layer.

\section{Conclusions}

In present study, electric field distribution, SAR and temperature variations are numerically analyzed in a twodimensional human abdomen model exposed to microwaves. The exposure frequency is $2450 \mathrm{MHz}$ with power densities 1 $\mathrm{mW} \mathrm{cm} \mathrm{cm} \mathrm{cm}^{-2}, 5 \mathrm{~mW}^{-2}$ and $50 \mathrm{~mW} \mathrm{~cm}^{-2}$. Electric field and local SAR have similar distribution pattern and their maximum values are determined in skin layer. The magnitude of electric field falls rapidly with linear distance, therefore, the electric field intensity and hence local SAR approach to zero within inner organs of abdomen. The maximum values of local SAR are above the ICNIRP safety limits for all the exposure power densities.

Pennes' BTE and thermal wave model TWMBT are employed to study the temperature changes in human trunk. Pennes' BTE assumes infinite speed of heat propagation inside the body while TWMBT is based on finite propagation speed. Hence, the temperature predicted by Pennes' BTE rises quickly and reaches steady state faster than that by TWMBT. Relaxation time is an important parameter in bioheat propagation; for greater values of relaxation time, temperature attains steady state more 
slowly. During transient state, there is significant difference between temperatures predicted by Pennes' BTE and thermal wave model TWMBT. Transient temperatures estimated by TWMBT are lower than that by Pennes' BTE This result is more important in the conditions where exposure duration is short.

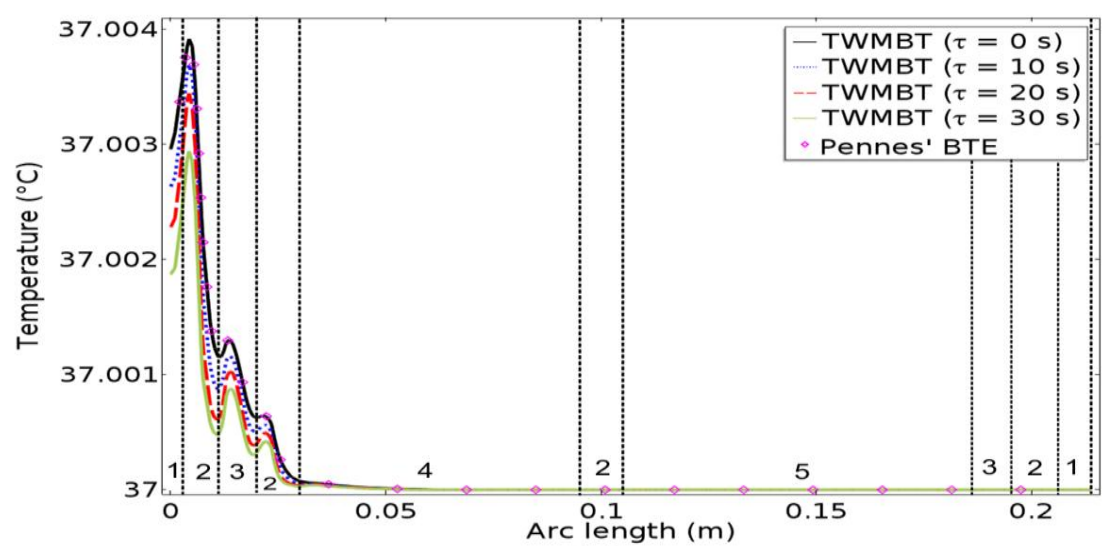

(a)

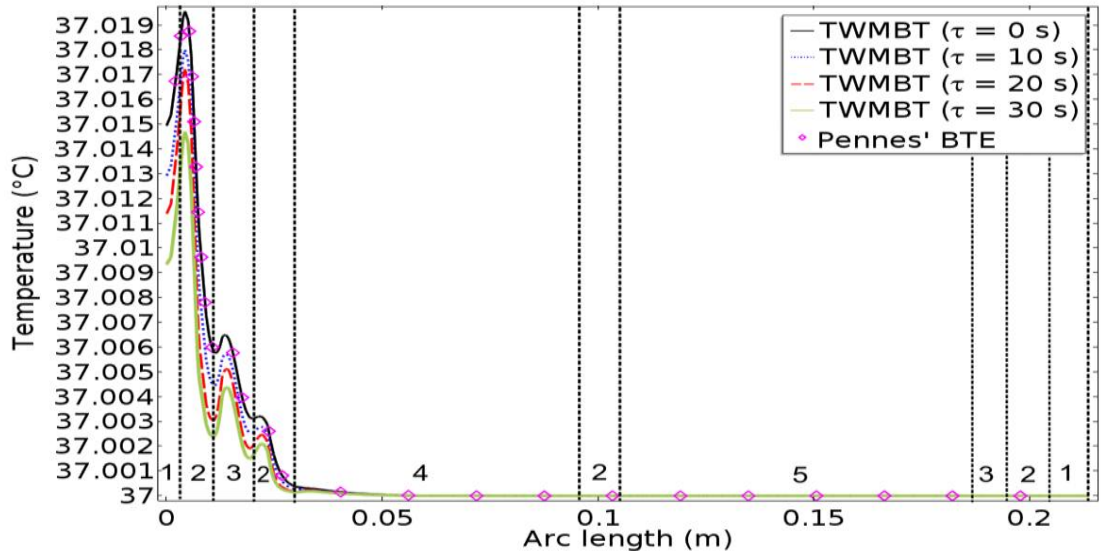

(b)

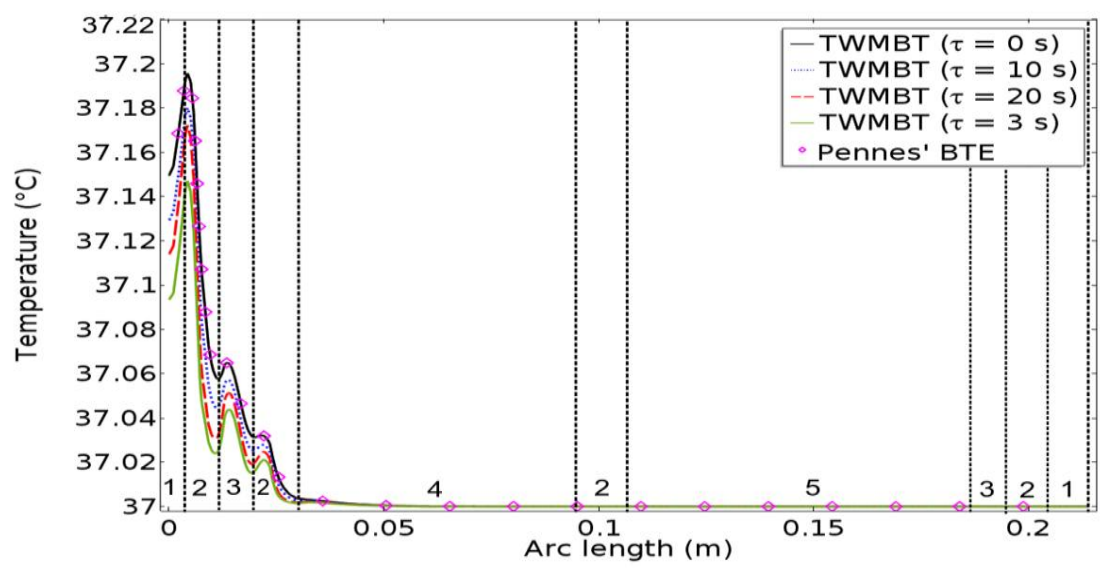

(c)

Figure 10: Temperature distribution at 1 minute of exposure for different relaxation times along the arc length at (a) $1 \mathrm{~mW} \mathrm{~cm}$, (b) $5 \mathrm{~mW} \mathrm{~cm}$ and (c) $50 \mathrm{~mW} \mathrm{~cm}^{-2}$. 1:skin, 2:fat, 3:muscle, 4: large intestine, 5:bone 


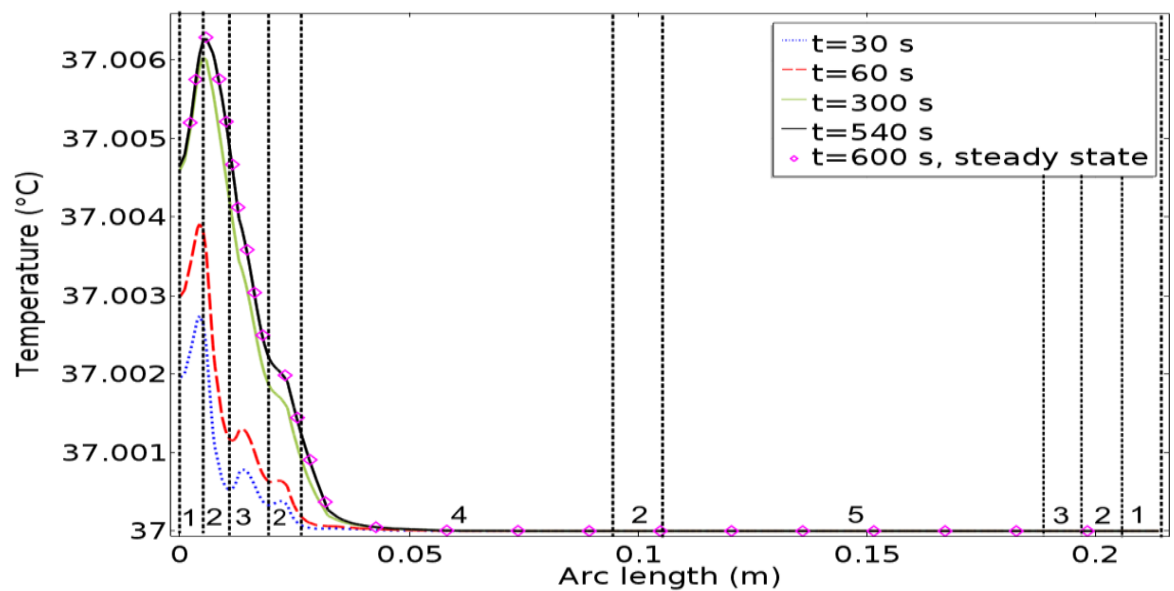

(a)

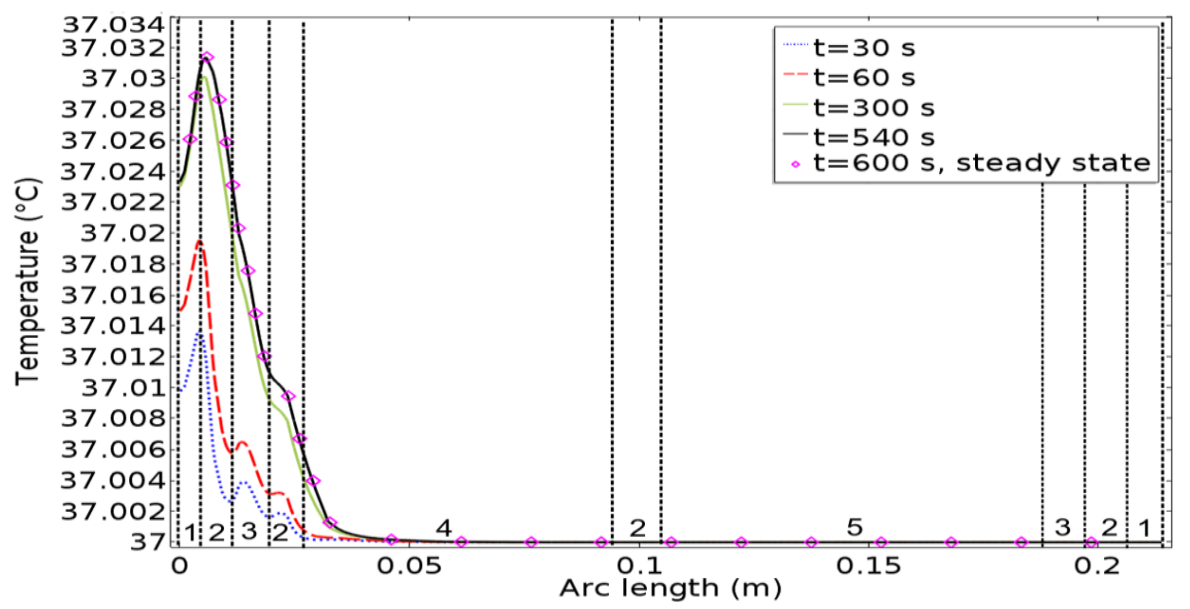

(b)

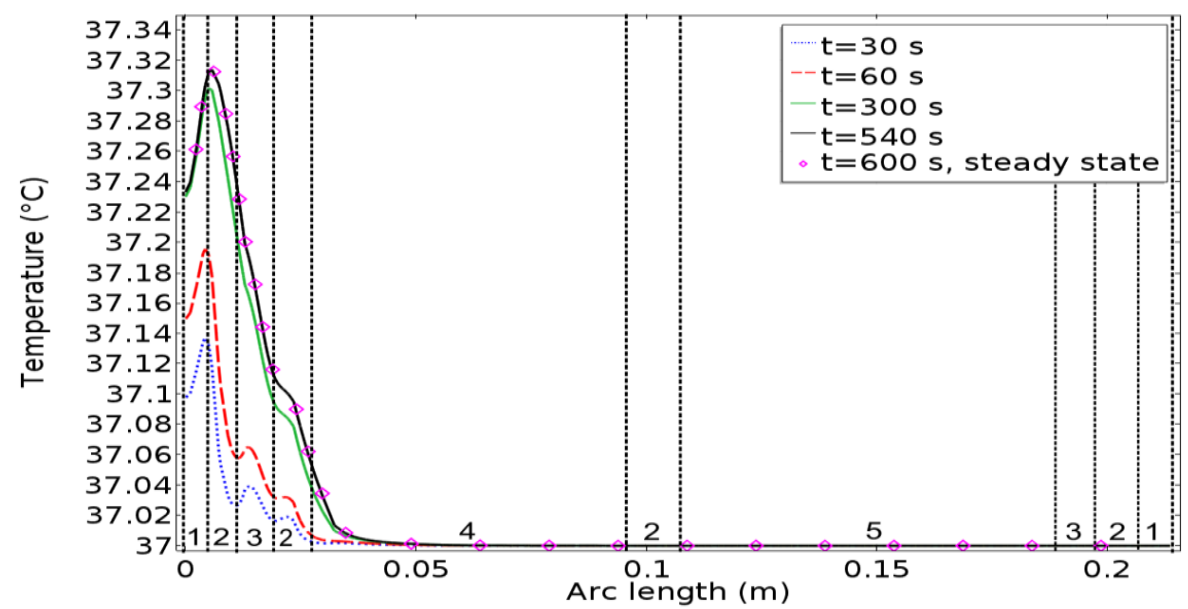

(c)

Figure 11: Temperature distribution at different times along arc length at (a) $1 \mathrm{~mW} \mathrm{~cm}^{-2}$, (b) $5 \mathrm{~mW} \mathrm{~cm}$ and (c) 50 $\mathrm{mW} \mathrm{cm}{ }^{-2} .1$ :skin, 2:fat, 3:muscle, 4: large intestine, 5:bone 
The rise in temperature depends directly on the power density of microwave exposure; for greater power density, temperature predicted is higher. Besides power density, other factors influencing temperature distribution includes relaxation time, blood perfusion, dielectric and thermal properties of tissues. This study may provide a detailed knowledge of thermal changes in human body exposed to microwave EM fields from various sources, since it explains the temperature variations estimated by traditional Pennes' BTE in addition to those by TWMBT. For more accurate and realistic assessment of electric field, SAR and thermal distribution, three-dimensional model of human body can be used for numerical analysis.

Conflict of Interest: The authors declare that they have no conflict of interest.

\section{References}

[1] R.F. Cleveland Jr, Radio frequency radiation in the environment: sources, exposure standard, and related issue. In: Carpenter DO, Ayrapetyan S, editors, Biological effects of electric and magnetic fields, Academic Press, New York, 1994.

[2] World Health Organization (WHO) and International Programme on Chemical Safety. (1993). Electromagnetic fields $(300 \mathrm{~Hz}$ to $300 \mathrm{GHz})$. Geneva: World Health Organization.

[3] K.1. Ryan, J.A. D'Andrea, J.R. Jauchem, P.A. Mason, Radio frequency radiation of millimeter wave length: potential occupational safety issues relating to surface heating, Health Phys 78(2): 170-81, 2000.

[4] International Commission on Non-Ionizing Radiation Protection (ICNIRP), Guidelines for limiting exposure to time-varying electric, magnetic and electromagnetic fields (up to $300 \mathrm{GHz})$, Health Phys. 74: 494-522, 1998.

[5] Federal Communications Commission (FCC), (2001) Evaluating Compliance with FCC Guidelines for Human Exposure to Radiofrequency Electromagnetic Fields, OET Bulletin 65, Supplement C, 01-01, 2001.

[6] IEEE, IEEE Standard for Safety Levels with Respect to Human Exposure to Radio Frequency Electromagnetic Fields, $3 \mathrm{kHz}$ to $300 \mathrm{GHz}$. IEEE Standard C95:1, 1999.

[7] C.H. Durney, H. Massoudi, F.I. Magdy, Radiofrequency radiation dosimetry handbook. 4th ed., Brooks Air Force Base; USAFSAM-TR-85-75, Texas, 1986.

[8] L.J. Challis, Mechanisms for interaction between RF fields and biological tissue, Bioelectromagnetics, Suppl 7: S98S106, 2005.

[9] M.A. Stuchly, Health Effects of Exposure to Electromagnetic Fields, IEEE Aerospace Applications Conference Proceedings, Aspen, CO, USA, pp.351-368, 1995.
[10] E.R. Adair, B.W.Adams, G.M. Akel, Minimal changes in hypothalamic temperature accompany microwave-induced alteration of thermoregulatory behavior, Bioelectromagnetics 5: 13-30, 1984 .

[11] H.H. Pennes, Analysis of Tissue and Arterial Blood Temperature in the Resting Human Forearm, Journal of Applied Physiology 1: 93-122,1948.

[12] U.D. Nguyen, J.S. Brown, I.A. Chang, J. Krycia, M.S Mirotznik, Numerical evaluation of heating of the human head due to magnetic resonance imaging, IEEE Trans Biomed Eng 51:1301-9, 2004.

[13] P. Bernardi, M. Cavagnaro, S. Pisa, E. Piuzzi, Specific absorption rate and temperature elevation in a subject exposed in the far-field of radio-frequency sources operating in the 10900-MHz range, IEEE Transactions on Biomedical Engineering 50: 295-304,2003.

[14] O.P. Gandhi, Q.X. Li, G. Kang, Temperature rise for the human head for cellular telephones and for peak SARs prescribed in safety guidelines, IEEE Transactions on Microwave Theory and Techniques 49:1607-1613,2001.

[15] O.P. Gandhi, G. Lazzi, C.M. Furse, Electromagnetic absorption in the human head and neck for mobile telephones at 835 and $1900 \mathrm{MHz}$, IEEE Trans. Microwave Theory Tech. 44: 1884-1897, 1996.

[16] T. Wessapan, S. Srisawatdhisukul, P. Rattanadecho, Specific Absorption Rate and Temperature Distributions in Human Head Subjected to Mobile Phone Radiation at Different Frequencies, Int. J. Heat Mass Transfer 55: 347-359, 2012.

[17] T. Wessapan, P. Rattanadecho, Specific absorption rate and temperature increase in the human eye due to electromagnetic fields exposure at different frequencies, International Journal of Heat and MassTransfer 64: 426-435, 2013.

[18] T. Wessapan, S. Srisawatdhisukul, P. Rattanadecho, Numerical analysis of specific absorption rate and heat transfer in the human body exposed to leakage electromagnetic field at $915 \mathrm{MHz}$ and $2450 \mathrm{MHz}$, Journal of Heat Transfer 133 : 051101, 2011.

[19] J. Liu, X. Chen, L.X. Xu, New thermal wave aspects on burn evaluation of skin subjected to instantaneous heating, IEEE Trans. Biomed. Eng. 46: 420-428, 1999.

[20] S. Ozen, S. Helhel, O. Cerezci, Heat analysis of biological tissue exposed to microwave by using thermal wave model of bio-heat transfer (TWMBT), Burns 34: 45-9, 2008.

[21] J. Liu, X. Zhang, C. Wang, W. Lu, Z. Ren, Generalized time delay bioheat equation and preliminary analysis on its wave nature, Chin. Sci. Bull. 42: 289-292, 1997. 
[22] F. Xu, T. Lu, K.A. Seffen, Dual-phase-lag model of skin bioheat transfer, Proceedings of the 2008 International Conference on BioMedical Engineering and Informatics, Sanya, China, pp. 505-511, 2008.

[23] K.C. Liu, Y.N. Wang, Y.S. Chen, Investigation on the bioheat transfer with the dual-phase-lag effect, International Journal of Thermal Sciences 58: 29-35, 2012.

[24] H. Ahmadikia, R. Fazlali, A. Moradi, Analytical solution of the parabolic and hyperbolic heat transfer equations with constant and transient heat flux conditions on skin tissue, International Communications in Heat and Mass Transfer 39: $121-130,2012$

[25] F. Xu, T. Lu, Analysis of skin bioheat transfer, in: Introduction to Skin Biothermomechanics and Thermal Pain, Springer, Berlin, Heidelberg, pp 69-83, 2011.

[26] F. Xu, K. Seffen, T. Lu, Non-Fourier analysis of skin biothermomechanics, International Journal of Heat and Mass Transfer 51: 2237-2259,2008.

[27] K. Mitra, S. Kumar, A. Vedevarz, M.K. Moallemi, Experimental evidence of hyperbolic heat conduction in processed meat, J. Heat Transfer 117: 568-573, 1995.

[28] A. Banerjee, A. Ogale, C. Das, K. Mitra, C. Subramanian, Temperature distribution in different materials due to short pulse laser irradiation, HeatTransfer Eng. 26: 41-49,2005.

[29] P. Antaki, New interpretation of non-Fourier heat conduction in processed meat, J. Heat Transfer 127(2): 189-193, 2005.

[30] W. Kaminski, Hyperbolic heat conduction equation for materials with a non homogeneous inner structure, J.Heat Transfer 112: 555-560, 1990.

[31] A.O. Rodrigues, J.J. Viana, L.O.C.Rodrigues, J.A. Ramirez, Calculation of temperature rise induced by cellular phones in the human head, J. Microwaves Optoelectron. 6: 310-322, 2007.

[32] H. Herwig, K. Beckert, Fourier versus non-fourier heat conduction in materials with a non homogeneous inner structure, J. Heat Transfer 122: 363-365, 1999.

[33] C. Cattaneo, A form of heat conduction equation which eliminates the paradox of instantaneous propagation, Сomp. Rend. 247: 431-433, 1958.

[34] P. Vernotte, Les paradoxes de la theorie continue de l'equation de la chaleur, Comp. Rend. 246: 3154-3155, 1958.

[35] K. Shiba, N. Higaki, Analysis of SAR and current density in human tissue surrounding an energy transmitting coil for a wireless capsule endoscope, Proceedings of the 20th International Zurich Symposium on Electromagnetic Compatibility, Zurich, pp. 321-324, 2009.

[36] R.L. Macintosh, V. Anderson, A comprehensive tissue properties database provided for the thermal assessment of a human at rest, Biophysical Reviews and Letters 05(03): 129-151, 2010.

[37] Gabriel C (1996) Compilation of the dielectric properties of body tissues at RF and microwave frequencies. Report N.AL/OE-TR- 1996-0037, Occupational and environmental health directorate, Radiofrequency Radiation Division, Brooks Air Force Base, Texas (USA).

[38] R.J. Spiegel, A review of numerical models for predicting the energy deposition and resultant thermal response of humans exposed to electromagnetic fields, IEEE Transactions on Microwave Theory and Techniques 32 (8): 730-746, 1984.

[39] S. Nishizawa, O. Hashimoto, Effectiveness analysis of lossy dielectric shields for a three-layered human model, IEEE Transactions on Microwave Theory and Techniques 47: 277$283,1999$. 